Check for updates

Cite this: Mater. Adv., 2021, 2, 2876

Received 27th January 2021, Accepted 5th April 2021

DOI: 10.1039/d1ma00069a

rsc.li/materials-advances

\section{Nano-enabled theranostics for cancer}

\author{
Palani Sharmiladevi, Koyeli Girigoswami, (D) Viswanathan Haribabu and \\ Agnishwar Girigoswami (D) *
}

\section{Introduction}

Life-threatening diseases like cancer cause 1 in 6 deaths globally and it was the second leading cause of death according to the report by the WHO in the year 2018. The incidence of cancer has rapidly increased over the years and continues to remain a threat. Cancer refers to the uncontrolled growth of cells that are typically insensitive to the anti-growth mechanism. These cancer cells possess the ability to replicate, evade apoptosis, form new blood vessels and undergo metastasis. Cancers are of many types but they have very limited common characteristics which make it difficult to treat them. ${ }^{1}$ Even though the incidence of cancer cannot be controlled, the mortality rate resulting from the disease can be reduced by early diagnosis. Imaging techniques used in early diagnosis are X-ray, endoscopy, ultrasound, magnetic resonance imaging (MRI), computed tomography (CT), positron emission tomography (PET), and single-photon emission tomography (SPECT). These techniques possess limitations such as low sensitivity and poor contrast between the benign and malignant lesions.

Tissues that are suspected to be abnormal by the imaging techniques such are MRI or CT are subjected to histopathology by taking a biopsy of the suspected tissue. Histopathology can help in diagnosing the tumour but not in the early stage. Thus, the development of an advanced tool for the early diagnosis of a disease like cancer is a major challenge and researchers have focused to address the limitations of the techniques that are being used in the current clinical settings.

Medical Bionanotechnolgy, Faculty of Allied Health Sciences, Chettinad Hospital and Research Institute (CHRI), Chettinad Academy of Research and Education (CARE), Kelambakkam, Chennai 603 103, India. E-mail: agnishwarg@gmail.com; Fax: +91044 4741 1011; Tel: +919445268 615

\section{Advantages of nanomaterials in molecular imaging}

The tremendous growth in molecular imaging techniques has led to the development of several new nanomaterials (Fig. 1). These novel nanomaterials are developed to tackle the critical issues faced by diagnostic imaging techniques. Imaging with nanomaterials offers significant advantages over the conventional approaches in several ways such as improved circulation times,

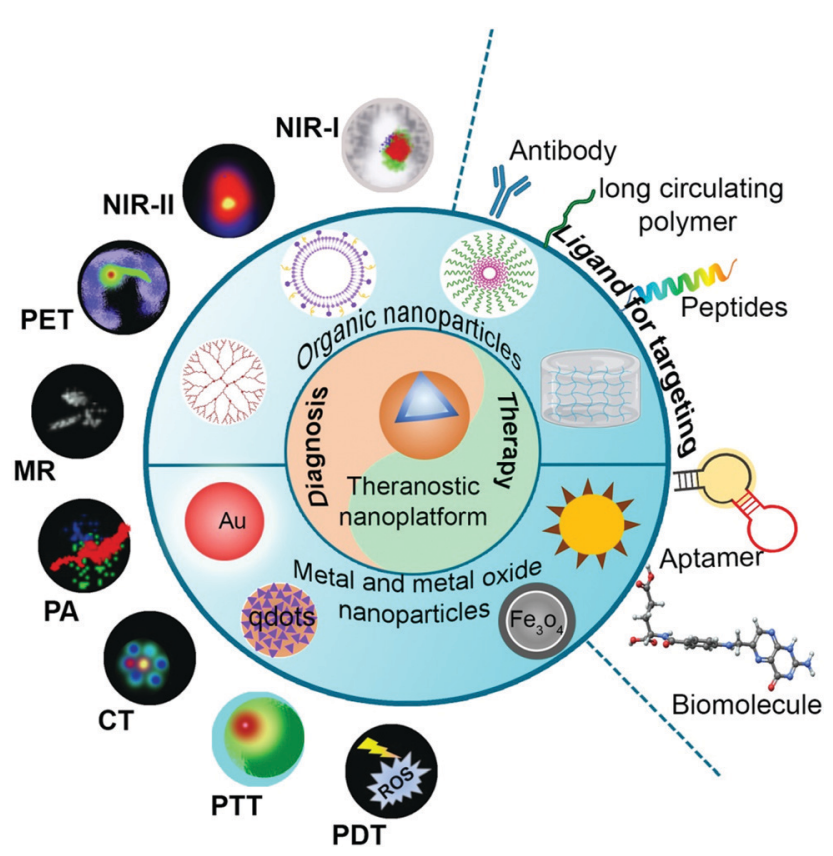

Fig. 1 Schematic illustration of the diagnostic and therapeutic applications of nanomaterials. 
high diagnostic specificity, and sensitivity. All the small molecules and nanomaterials are to be extensively analyzed for their desired outcomes and risks before selecting for application.

Nanomaterials are considered to be good candidates for several applications because of their (i) small size (ii) effective target binding (iii) highly controllable physicochemical properties (iv) high payload delivery (v) potentiality to be complexed with multiple imaging modalities and (vi) the potential for payload combination with therapeutics, e.g., theranostic agents. ${ }^{2}$

The primary goal of non-invasive imaging is not only to visualize the physiology but also to measure and localize the molecular targets and pathways that are specific to a disease state. Contrast based imaging allows identifying the disease site by getting accumulated in the disease site and produces a signal that can be identified by the imaging modality. ${ }^{3-5}$ Traditionally, these contrast agents are administered systematically and are mainly composed of molecules, peptides, antibodies, aptamers and proteins. These agents are specifically designed to get landed in the disease site by utilizing the fundamental biological differences between healthy and diseased tissues.

To develop new molecules to image the diseases, an interdisciplinary team of chemists, clinicians, biologists, and engineers are working together. These teams prefer nanomaterials over the small molecules, proteins, and peptide-based strategies. The possible reason is, like nanomaterials, it is not possible to engineer the small molecules to carry high payloads or to combine with drugs (e.g., theranostics). Small molecules can travel within the body and can also easily enter the cancer cells. Therefore, nanomaterials have to be non-toxic with high sensitivity and resolution in imaging in order to compete with the small molecules.

Owing to their long history small molecules are still being preferred over nanomaterials in the clinical settings for imaging. This fact shows that nanomaterials have to overcome certain limitations to be clinically translated.

Imaging modalities provides the ability to visualize the anatomical and physiological details along with the functional phenomena associated with the disease. In most cases, anatomical and functional imaging is used in parallel. While anatomical imaging reveals the physical structures of the body whereas functional imaging helps to identify the physiological and biochemical activities. ${ }^{6}$ Imaging modalities have long been used by physicians to identify the pathology with precise details such as its exact location and size. In recent times, molecular imaging along with functional imaging has helped to diagnose and understand the changes occurring at the physiological level to assess the severity and molecular phenotype of the disease conditions such as heart diseases, cancer, and several others. ${ }^{7}$

\subsection{Essential properties of nanomaterials}

Nanomaterials to be used as an imaging agent requires a suitable structure along with a biocompatible coating to reduce the toxic nature, improve its pharmacological parameters, and to serve as a linker to attach drug moieties and imaging agents. The properties of imageable nanomaterials are dependent on their physicochemical properties. Toxicity is a major concern with nanomaterials and that should be kept under check to use them for in vivo imaging, ${ }^{4,8-10}$ as these contrast agents will be directly injected into healthy populations for screening the presence of diseases. Thus, nanomaterials should not possess any toxic effects.

Nanomaterials should possess the ability to get accumulated in a specific site within a specific time. In the case of cancer screening, these nanomaterials have to be engineered with a certain size and shape so that they will be able to extravasate through the EPR (enhanced permeability and retention) effect. ${ }^{11}$ Blood pool agents are contrast agents that are specifically designed to map the blood vessels. Hence, nanomaterials intended for use in mapping the blood vessels should have longer circulation times. ${ }^{12}$

Stability is an important prerequisite of nanomaterials so that the signal will be continuous during the imaging procedure and at the same time these particles should also be able to get cleared from the system. Many other characteristics govern the in vivo behavior of the nanomaterials and thus have a significant effect on the imaging properties as well. ${ }^{13}$ One such feature is the polydispersity that has great importance. Polydispersity represents the whole distribution of size in a given nanoformulation. Aggregation among the nanomaterials also affects polydispersity. Polydispersity is usually controlled by the synthesis mechanism, type of solvent used, and charge of the nanoparticles. Interestingly aggregation of the nanomaterials may lead to positive or negative effects on the imaging ability depending on the context.

Mostly, aggregation of the nanomaterials does not have a good effect on the biodistribution properties. Aggregation increases the hydrodynamic diameter of the nanoparticles and thus severely affects the circulation times, distribution in the tissue site, capillary blockage and also causes the nanoparticles to be captured by the mononuclear phagocyte system (MPS). ${ }^{14}$ Aggregation also increases the MPS uptake which in turn reduces the availability of the nanomaterials to get landed in the target site. ${ }^{14}$ Similarly, aggregation can also lead to decreased signal intensities in platforms such as magnetic particle imaging (MPI). For instance, when iron oxide nanoparticles get aggregated, it leads to considerable variation in their magnetic behavior thus reducing its signal in MPI as this technique is primarily based on the detection of magnetic moments. ${ }^{15}$ Recent studies have also demonstrated the relationship between the polydispersity of the nanomaterials and the protein corona. Protein corona is defined as the protein cloud that gets adsorbed on the surface of the nanomaterial upon its exposure to fluids such as blood present in the in vivo system. This protein adsorption has been found to influence the biodistribution and tracking of the path of the nanoparticle in vivo. ${ }^{16}$

Some studies also suggest that protein corona can be used as a major predictive feature to determine the nanoparticles' in vivo behavior. This was demonstrated in a protein corona study done with the same base polystyrene with different surface functionalization which showed the influence of protein corona in the aggregation of the nanoparticles concerning the surface 
chemistry (e.g., charge). This aggregation substantially influenced the nanoparticles biodistribution nature. Even though the administration of the particles by intravenous injection resulted in the uptake of the particles in the in vivo system, their biodistribution was found to be dependent on the formation of the protein corona. In this case, few nanomaterials got accumulated in the lungs and few were found in the skin. ${ }^{17}$

The emergence of nano-based contrast agents for imaging arose in demand for improving the contrast of the MR images in vivo. As iron oxide nanoparticles were in clinical use for the treatment of anaemia since the 1960s, utilizing the nanoparticles for imaging applications turned a natural fit. ${ }^{18}$

\subsection{Limitations of nanomaterials}

Despite the many advantages offered by nanomaterials, still, some limitations need to be addressed. And in certain cases, the major advantage of the nanomaterials turns out to be a greater disadvantage too. For example, in certain cases, the size of the nanomaterials may be larger to get accumulated in the desired tissues. ${ }^{11}$ This can be overcome by optimizing parameters such as charge and functionalization.

Nanomaterials must also possess the ability to get cleared from the system. While small molecules are capable of either getting metabolized or cleared from the system, but in some cases, the makeup of the nanoparticle is advantageous as it can be useful to the body. One good example is the use of iron oxide nanomaterials. These nanomaterials help in imaging and also get degraded (e.g., for anaemic patients) in the system thereby increasing the body's iron stores.

In other cases, the fundamental makeup in itself acts as a toxic material. Quantum dots made up of heavy metals such as selenium, cadmium, and arsenic are not much preferred for clinical translation as their degradation products will be highly toxic, nevertheless, they could be fabricated in a way that will help to get them cleared via renal or hepatobiliary excretion. ${ }^{19}$

\subsection{The superiority of MRI in the diagnosis of cancer}

The non-invasive nature along with high spatial resolution has made MRI an important tool in the diagnosis of diseases and has been widely employed in preclinical researches. Research in the development of novel imaging probes and methods to detect the molecular signatures has strengthened MRI in characterizing the molecular and physiological changes occurring in the tissues. Altered cellular metabolism is an essential factor for the growth and spreading of cancer. ${ }^{20}$ Several biochemical pathways such as pentose phosphate pathway (PPP), TCA cycle, and glycolysis undergo alternative regulation in cancer cells. ${ }^{21}$ Monitoring and understanding these changes is essential to diagnose and plan cancer treatment. Based on Otto Warburg's finding that tumor and normal cells have different metabolism rates different MR imaging techniques were developed to monitor the metabolic changes occurring in the cancer tissues.

2.3.1 Magnetic resonance spectroscopy (MRS). This technique is employed widely to detect metabolic changes in both normal as well as in cancerous tissues. ${ }^{22}$ The markers that are detectable by MRS are helpful in understanding and differentiating the metabolic tumor phenotypes. Proton MRS $\left({ }^{1} \mathrm{H}\right.$ MRS) is a widely used MRS technique to detect the metabolic changes occurring in the cancer tissues. ${ }^{23}$

2.3.2 Hyperpolarized MRI in cancer. This technique uses hyperpolarized ${ }^{13} \mathrm{C}$ labeled pyruvate to assess the level of glycolysis that takes place in cancerous tissues. Thus, this technique holds the potential to use MRI for improved cancer detection and characterization. ${ }^{13} \mathrm{C}$ is the most commonly used hyperpolarized substrate in clinical and preclinical studies. ${ }^{24}$ This technique also demonstrated the ability to map the $\mathrm{pH}$ of the tissue. ${ }^{25}$ As it is well known that changes in $\mathrm{pH}$ are associated with tissue pathology, monitoring these changes would help in assessing the pathological state of the tissue.

2.3.3 Chemical exchange saturation transfer (CEST) imaging. CEST is a new contrast enhancement technique that allows to indirectly monitor the molecules and macromolecules that possess exchangeable protons. In this technique, the exchangeable water protons are selectively saturated with a radiofrequency (RF) saturation pulse which reduces saturation magnetization of the protons and thus decreases the bulk water signal. ${ }^{26}$ The decreased bulk water signal is quantified and mapped to obtain highresolution images of selected molecules and macromolecules.

2.3.4 Imaging of peptides and mobile proteins. Mobile protein and peptides in cancer are monitored by Amide proton transfer (APT) imaging. This is one of the most widely used CEST based methods and depends on the exchange between water molecule protons and the amide groups present in the proteins and peptides. High numbers of amide protons are reported to be found in cancer tissues in comparison to normal tissues. It has been demonstrated that APT imaging has shown superiority to differentiate tumor from oedema and normal brain area, and to identify the high grade and low-grade tumors. $^{27}$

2.3.5 Identification of reporter genes. Ex vivo examination methods such as immunohistochemistry and histology are used to examine the reporter genes such as luciferase and beta-galactosidase. Monitoring of these reporter genes allows studying gene expression in cancer cells. The employed ex vivo methods can provide specific and sensitive information, but they are not capable of providing dynamic information. Optical imaging, on the other hand, is also capable of producing structural and functional information about the expression of genes in vivo but has limited sensitivity. The utilization of MR based reporter genes has not only helped to overcome these issues but also enabled to monitor the process of cell proliferation and migration in vivo.

2.3.6 Cancer immunotherapy and MR imaging. Cell-based cancer immunotherapy is gaining importance as radiotherapy and chemotherapy are not sufficient enough to fight against cancer. This treatment method triggers the immune system of the patients to induce an anti-tumor response. Immunotherapy is efficacious and can selectively target the cancer cells and thus reduces the severity of side effects. Non-invasive monitoring of the biodistribution of these cells will help to develop effective cell-based therapy. With the help of non-invasive imaging, the fate of these cells can be monitored. To non-invasively probe 
these therapeutic cells, imaging probes that are detectable by MRI are conjugated on their surface before in vivo administration. Thus, by assessing the imaging signals it is possible to differentiate the patients as responders and non-responders to immunotherapy. Labelling the therapeutic cells with nanoparticles holds the advantage of being translated clinically as nanoparticles significantly improved the hypointense contrast on MRI in a study by Sheu et $a .^{28}$

\section{Contrast mechanisms}

MR image is said to have contrast if it has variations in signal levels. High signal areas appear as bright whereas the low signal areas appear as dark. And the areas with intermediate signal appear as grey shades. The NMV associated with a tissue can be separated into individual vectors such as fat, muscle, and cerebrospinal fluid (CSF). MRI contrast is mainly obtained through the recovery and decay of $T_{1}$ and $T_{2}$ mechanisms and proton or spin density. The $T_{1}$ and $T_{2}$ relaxation mechanisms are dependent on the inherent energy levels of the tissues, the package of the molecules, and the matching of the tumbling rate with the Larmor frequency of hydrogen.

\subsection{Relaxation in different tissues}

There are two extremes of contrast in MRI and they are fat and water. Fat contains molecules of lipids with a very slow molecular tumbling rate because of the large fat molecules consisting of hydrogen-bonded to carbon and oxygen.

Whereas in water molecules the hydrogen is bonded with only one oxygen atom which paves more spacing between the molecules and hence the molecules tumbles at a faster rate than the fat molecules. Thus, hydrogen has high Larmor frequency in water than in fat. And in the recovery process fat has rapid recovery than water. Hence fat and water molecules appear with different contrast in MR images.

\subsection{Contrast agents in MRI}

In MRI the image contrast is determined by the relaxation mechanisms. Different tissues have different relaxation times and hence the images appear differently from each other. Contrast agents used in MRI can affect the $T_{1}$ and $T_{2}$ relaxation times by affecting the local magnetic field. This is the reason why MRI contrast agents have varying magnetic susceptibilities. Gadolinium (Gd) based contrast agents are most commonly used in MRI, and is usually chelated or bound to other molecules to reduce their toxicity. Gd is paramagnetic at the normal body temperature and exhibits a positive effect over the local magnetic field.

Gd agents can shorten the $T_{1}$ relaxation time by exhibiting a positive effect on the magnetic field and thus can produce brighter images of the tissues in $T_{1}$ weighted images. The contrast agent (CA) that generates bright or positive contrast is known as a $T_{1}$ contrast agent and that generates negative or dark contrast is known as a $T_{2}$ contrast agent. Gadolinium CAs can affect the relaxation times of both $T_{1}$ and $T_{2}$ thereby shortening both $T_{1}$ and $T_{2}$. CAs that have a pronounced effect on $T_{1}$ weighted images are known as $T_{1}$ CAs and those that exhibit the same on $T_{2}$ weighted images are known as $T_{2}$ CAs. The $T_{2}$ effects of Gd are much smaller than the $T_{1}$ and hence Gd is primarily used as $T_{1}$ CA.

\section{3 $T_{1}$ contrast agents}

The tumbling rate of water molecules exceeds that of the Larmor frequency and results in long $T_{1}$ relaxation time and inefficient relaxation. This causes the water molecules to appear as dark images in $T_{1}$ weighted imaging. When a molecule with a large magnetic moment undergoes tumbling near the water protons it causes fluctuations in the local magnetic field. Gadolinium chelates in the vicinity of water protons create fluctuations in the local magnetic field, which are near the Larmor frequency. Thus Gd helps in shortening the relaxation times of the water molecules and produces positive contrast images. The same effect occurs with any contrast agent that has larger magnetic moments.

Owing to the positive magnetic susceptibilities, paramagnetic substances are a suitable choice to act as a contrast agent in MRI. Gadolinium is considered an ideal contrast agent because of its electronic configuration which holds seven unpaired electrons that favors rapid water exchange.

The magnetic moment $(\mu)$ of an unpaired electron is 500000 times larger than that of hydrogen proton and thus causes fluctuations in the local magnetic field. Even though it is possible to obtain images of some lesions without the administration of the contrast agents, it is not possible to visualize all lesions without the contrast agents.

\section{$3.4 \quad T_{2}$ contrast agents}

As mentioned earlier, contrast agents can affect both the $T_{1}$ and $T_{2}$ relaxation times of the water protons, but are classified as $T_{1}$ or $T_{2}$ contrast agent depending on its pronounced effect on either one of the relaxation times. Iron oxide nanoparticles can affect both the relaxation times but has a higher effect on $T_{2}$ than $T_{1}$ because the strong magnetic field causes rapid dephasing of the nuclei. Thus causing notable signal attenuation and the ratio of $r_{2} / r_{1}$ is greater than 10 . Thus iron oxide nanoparticles are considered as $T_{2}$ contrast agents. $T_{2}$ contrast agents can exhibit a strong contrast effect and it's mainly dependent on the magnetic moment of the nanoparticles. Even though $T_{2}$ contrast agents can generate excellent contrast they are prone to be confused with other endogenous conditions that may lead to misinterpretations. Hence $T_{1}$ contrast agents remain the choice of the clinicians.

\subsection{High relaxivity agents and their safety}

The amount of gadolinium present in the contrast agent determines its relaxivity properties. And newer gadolinium contrast agents were manufactured with higher relaxivity for a lower dose. These high relaxivity agents were designed to provide much better visualization of the pathologies with lower doses. One such FDA-approved high relaxivity agent is Gd-BOPTA which has the advantage of providing a higher signal from tiny vessels in MRA and high lesion complicity. 
Heavy metals are known to be toxic to humans and so is gadolinium. It's a toxic rare earth metal and could act as a cumulative poison in its native state. These metal ions tend to get accumulated within the sites of the body such as the liver, spleen, lungs, bone, membranes, enzymes, and transport proteins.

To overcome this accumulation of the gadolinium ions in the body, these agents were coupled with the chelating agents. Chelates are substances with a very high affinity for binding metal ions. Hence, chelates were used to bind the metal ions of gadolinium so that the toxic effects can be minimized to a great extent. Gadolinium was chelated with diethylenetriaminepentaacetic acid (DTPA) and Gd-DTPA was the first effective chelate to demonstrate as effective MR contrast media. When gadolinium is bound with a chelate few sites are left free to facilitate the water exchange process. In the case of DTPA, of the nine binding sites, eight is bound with the chelate and the ninth site is left free for interacting with the water protons.

The binding of the gadolinium ion to the chelates not only reduced the toxicity but also aided in the rapid excretion of the agent by the body. It is also to be noted that the biological half-life of gadolinium inside the human body is less than 2 hours only under the condition of a normal renal function. This time is prolonged in patients with impaired renal function. A wide number of paramagnetic gadolinium-based contrast agents have been approved for use and each agent varies with the chelate used to bind the heavy metal ion. Chelates can be of ionic, non-ionic, linear, and macrocyclic molecules. Another safety concern is the stability of the gadolinium chelates i.e. if the chelate is not stable gadolinium can easily come out from the chelate which will leave the gadolinium inside the system leading to adverse side effects.

\subsection{Nephrogenic systemic fibrosis}

The use of gadolinium agents was considered safe until a study conducted in 2006 raised health concerns over the use of these agents in patients with renal insufficiency for MRI and MRA procedures. Patients with renal insufficiency were not able to excrete these agents and its accumulation leads them to contradict nephrogenic systemic fibrosis (NSF). It is a condition that causes a 'bark-like' skin and later affects the whole organ system. NSF is a serious condition as there is no cure available yet. The condition may be treated earlier if diagnosed at the earliest. But this is very unlikely to happen as NSF takes several days to weeks to reveal the symptoms after the administration of the agent. Hence, a gadolinium contrast agent is a contraindication and requires precaution and care to be used in the clinical setting.

\subsection{Magnetic nanomaterials as alternative contrast agents}

Elements such as manganese and iron oxide are widely being researched for their potential to act as contrast agents in MRI. ${ }^{29}$ Manganese based contrast agents are used for liver imaging in which they are taken up by the kupffer cells. The normal liver thus produces a bright image and the lesions remain darker.

Iron-based nanoparticles are the most researched magnetic nanomaterials and have demonstrated safe use in humans for a long time. Several methods have been developed to synthesize iron oxide nanoparticles with different shapes and sizes and also have been successfully combined with other materials. Among them, iron oxide nanoparticles are the most preferred ones. The knowledge of nanochemistry has been extensively applied in the fabrication of iron and all other magnetic nanomaterials. This led to the synthesis of new materials with high saturation magnetization, magnetic moments, and coercivity for improved contrast in imaging based on magnetic nanoparticles.

Starting from doped iron oxides (e.g., Mn, Co, and Zn), ironcobalt (FeCo), iron carbides $\left(\mathrm{Fe}_{5} \mathrm{C}_{2}\right)$ to amorphous iron, newly developed magnetic nanomaterials are in the beginning to transform into the preclinical application by improving the image contrast and decreasing the amount required to achieve it. The fabrication chemistries of the iron oxide nanomaterials have been redesigned to improve their properties.

Several research groups have come up with novel strategies such as decreasing the non-specific binding and improving the stability of the particle across a wide range of salinity, $\mathrm{pH}$ to reach a higher relaxivity value for obtaining an optimized signal in MRI. ${ }^{30}$

Magnetic nanomaterials were primarily used in the domain of MRI which still continues. Recent technological advances have paved ways to exploit the magnetic nanomaterials for techniques such as magnetic particle imaging (MPI), magnetomotive photo-acoustics (MMPA), magneto-motive ultrasound (MMUS), and magneto-motive optical coherence tomography (MMOCT). These imaging modalities are in their nascent stage with few research groups working on the in vivo imaging application and the results obtained so far seems highly promising. These modalities are intensely related to the magnetic properties of the nanomaterials and cannot function like MRI even without using contrast agents.

Hence, it is necessary to optimize the magnetic properties of the samples in accordance with the need of the imaging modality in which it will be used. For example, MRI requires the interaction of the magnetic moments with the molecules present in the local environment whereas some modalities like magnetic particle imaging and magneto-motive imaging require the direct interaction of the nanomaterials with the applied magnetic fields.

Even though the physics of MPI and MRI is different from each other, they employ similar concepts of the pulse sequences, magnetic excitation, relaxation, and detection. MPI allows to quantitatively map magnetic nanomaterials 3D spatial distributions because unlike MRI there are no endogenous materials that can interfere with the signal. ${ }^{31}$

Magneto-motive approaches are primarily based on the application of a pulsed magnetic field to magnetic nanoparticles and monitoring the motions (i.e., displacements) induced by them. To track these displacements several strategies have been developed and are linked to other magneto-responsive imaging modalities. The use of magnetic nanomaterials in these imaging modalities allows expanding the window of imaging tissues that were once not possible because of the larger size of the contrast agents. ${ }^{10}$ 


\section{Conventional cancer therapies}

Humans' quest for the design of effective treatment is neverending. Over the years, knowledge about the function and composition of the various components of the human body (muscles, nerves, organs, bones, blood vessels) and importantly the blood cells which are the main building blocks has improved a lot.

This understanding has led to the design of a new range of medications including both natural and synthetic to fight against systematic (either physical or mental) disorders, infectious diseases, damage and malfunction to the components of the body, and inflammatory illnesses. This demand for the development of new drugs reaches its peak particularly during the emergence of new diseases/disorders that pose a global risk to the human population and when drugs fail to treat the bacterial or viral infections due to the development of resistance mechanisms in the microorganisms. Cancer is one such global risk that challenges the mortality of humans to a greater extent.

Effective cancer therapy requires detailed knowledge about the pathophysiology of cancer, novel anti-cancer drugs, and biomedical technologies. The development of cancer therapy has brought scientists from different areas of expertise such as materials science, oncology, molecular biology, chemistry, and biomedicine to work together. Thus cancer research has evolved into an area of interdisciplinary research.

Below listed are the most commonly used treatment procedures for treating cancer.

1. Radiation therapy.

2. Surgery.

3. Cytotoxic chemotherapy.

\subsection{Radiation therapy}

Radiation therapy aims to kill the cancer cells at a specific region of the body by the application of high-intensity energy beams. It works by producing small breaks in DNA and thus prevents cancer cells from rapid proliferation. Unlike chemotherapy in which the healthy cells are exposed to the cytotoxic drugs, radiation therapy is specifically localized to the tumor site with the aim of maximum damage to the cancer cells and little harm to the surrounding healthy tissues. ${ }^{32}$

The major goals of radiation therapy are:

(i) To cure or shrink early-stage cancer:

Certain types of cancer are sensitive to radiation and thus radiation therapy causes the cancer cells to shrink. In a few cases, the patient is given a few cycles of chemotherapy and then subjected to radiation therapy. Radiation therapy is also performed before surgery to reduce the size of the tumor or after surgery to prevent its recurrence. Radiation therapy is also combined with chemotherapy as certain chemo drugs are capable of acting as radiosensitizers which will increase the sensitivity of the cancer cells to radiation. The major drawback of combining chemotherapy and radiation therapy is the risk of severe side effects. ${ }^{33}$

(ii) To stop recurrence in other areas:

Radiation therapy is done to prevent the recurrence of tumors. In the case of patients suffering from lung cancer, they are treated with radiation in the head region as this type of cancer poses a high risk of affecting the brain. In case of the reduced distance between existing cancer and the prevention area radiation therapy can be applied to both areas at the same time.

(iii) To treat the symptoms caused by advanced cancer:

Advanced stages of cancer can cause severe complications in that case radiation therapy might help to relieve the symptoms of pain, bowel blockages, and trouble in breathing or swallowing. The application of radiation therapy to treat the symptoms caused by advanced-stage cancer is known as palliative radiation therapy. ${ }^{34}$

4.1.1 Limitations. - Radiation therapy can also slightly increase the risk of occurrence of cancer at a nearby site due to the use of ionizing radiation.

- Radiation therapy is harmful to the foetus hence the women undergoing radiotherapy should not become pregnant during the entire duration of the treatment.

- It is not effective in treating the tumors that have started to metastasize.

4.1.2 Side effects. Radiation therapy specifically kills the cancer cells but also has the potential to damage the healthy cells which can cause several side effects. Side effects may occur during the treatment or after a few days or even weeks. Most side effects start to fade within weeks to two months after the completion of the treatment. In case of high dosage, cells may not be able to undergo self-repair and hence the side effects may last for a longer time. Generally, the side effects depend on the.

1. Type of radiation that was used.

2. The part of the body being treated.

3 . The dose of radiation and duration of the treatment.

4. The health condition of the patient.

The most common side effects of radiation therapy are fatigue, skin problems, hair loss, loss of appetite, nausea, and vomiting, and low blood cell count. ${ }^{35}$

Side effects of radiation therapy based on different organs under treatment are listed below in the table.

\subsection{Cancer surgery}

Surgery is used as a part of the cancer treatment plan in the following ways.

To prevent cancer: Tissues that are at high risk of becoming cancerous are removed by surgery. ${ }^{36}$ This type of surgery to prevent or lower the risk of occurrence of the tumor is known as preventive or prophylactic surgery.

(i) To diagnose and stage cancer:

Different types of surgical procedures are used to diagnose and stage cancer. Through the invasive procedure, a small tissue sample is collected and examined to check the presence of cancer cells. Further removal of a tumor by surgery helps to formulate the design plan. Examination of the biopsy sample reveals the exact type and grade of cancer and also the presence of tumor in the lymph nodes.

(ii) Removal of cancer:

The main goal of cancer surgery is to completely remove the tumor from the specific region of the body. Cancer surgery is 
effective in the case of localized tumors that haven't started to metastasize. During cancer surgery, a small amount of the surrounding normal tissue is also removed (surgical margin) to avoid the recurrence of the tumor. The amount of additional tissue removal depends on the type of tumor and its location inside the body. Depending on the type of tumor lymph nodes that are closer to the tumor may also be removed as they possess high chances of having cancer cells in them. If left unchecked the cancer cells present in the lymph nodes will form new tumors or may spread to some other parts of the body.

In certain cases even though it is not possible to remove the tumor completely still surgeons perform surgery to create a bypass around a blocked organ which will help to relieve the pain caused by the tumor. This type of surgery is called palliative surgery.

4.2.1 Limitations. Sometimes it is not possible to completely remove cancer because of the smaller size of the tumor that is not possible to see by the surgeon. The location of the tumor is also a deciding factor in opting for surgery as the removal of the tumor should not cause any damage to the nearby organs. The general health of the person is also to be considered before surgery. ${ }^{37}$

4.2.2 Side effects. Similar to other cancer treatments surgery also has risks, benefits, and side effects. The severity of the effects varies from one person to another and is also based on several factors such as the type of cancer, its location, and the treatments undergone before surgery such as radiation therapy or chemotherapy. The most common side effects of surgery are listed below:

Pain. This is a common side effect after surgery and the amount of pain depends on the part of the body where the surgery was performed, the size of the incision, and the amount of tissue removed.

Fatigue. Fatigue after surgery is more common in patients who had surgery in the abdomen or stomach. Fatigue is caused by stress, administration of anaesthesia, and loss of appetite.

The other side effects of surgery are loss of appetite, swelling, fluid accumulation and drainage from the surgery site, bleeding, infection, numbness, lymphedema, and organ dysfunction. The other concerns after surgery are dietary concerns, body image, fertility, and sexual side effects. ${ }^{34}$

\subsection{Chemotherapy}

Radiotherapy and surgery are the most preferred modes to treat localized solid tumors but are not effective in cases of metastasis and recurrence at local and distant sites. Cytotoxic chemotherapy is much sought to prolong the life expectancy of cancer patients in the advanced stage.

In the case of solid tumors chemotherapy often results in the reduction of the tumor volume and thus prolongs the survival rate of the patient in comparison to the natural course of the tumor. Cancer cells develop resistance in several cases and lead to the failure of the treatment and in the cases of intrinsic and acquired mutations the tumor cells are protected from the chemotherapy drugs by tumorigenesis. ${ }^{38}$

Chemotherapy is also used as an adjuvant therapy to patients with a high risk of recurrence after undergoing surgery. Neoadjuvant chemotherapy is done for the treatment of localized tumors before surgery. Opting to treat the tumor with neoadjuvant chemotherapy before the planned surgery has been shown to improve the survival rate of the patients undergoing surgery in cases of oesophageal cancer. In certain cases, chemotherapy is combined with radiotherapy to improve the effectiveness of the treatment.

\subsubsection{Limitations}

(i) Non-selectivity. Both the normal and cancer cells are governed by common cell proliferation processes and hence are susceptible to damage by the anti-cancer drugs. However, selectivity is observed in certain types of cancer as they demonstrate higher proliferation rates as in the case of highgrade lymphoma. The principle aim of chemotherapy is to achieve maximum killing of the cancer cells with minimum damage to normal tissues.

(ii) Delivery of chemotherapy. Anticancer drugs are administered by various routes depending on the pharmacological properties of the drug such as half-life, metabolism, and absorption. Most anticancer drugs are poorly soluble hence they are administered intravenously. Even though the use of oral anticancer drugs is convenient, they offer poor compliance and risk of unintentional overdose. Hence it is important to ensure the safety of this route of administration. Chemotherapy is also administered through the intrathecal route in the cases of confirmed meningeal disease and certain haematological implications. Strict guidelines have been implemented in certain countries to reduce the risk of error and ensure the safety of patients undergoing intrathecal drugs.

\subsubsection{Side effects}

(i) Acute complications. The most sensitive tissues of the human body are bone marrow, gonads, gastrointestinal mucosa, and hair follicles where the cells undergo rapid cell division. Thus, the immediate side effects of the chemotherapy cause myelosuppression, nausea, hair loss, vomiting, and reduced fertility. The possibility of fertility recovery is based on the age of the patient, dose and type of the drug administered, and duration of the treatment. The side effects of the chemotherapy are not only cytotoxic to spermatogenesis and oogenesis they are also teratogenic and harmful to the foetus. Hence patients to be treated with the chemotherapeutic drugs are counselled before the treatment to use appropriate contraception during the entire duration of the treatment.

Administration of chemotherapy causes two critical issues in patients. They are infection and occurrence of necrosis and blisters during the administration of the drug. Cytotoxic chemotherapy will be the major treatment option and the problem of chemoresistance and toxicity complications needs to be overcome.

(ii) Late effects of chemotherapy. Chemotherapy can cause direct side effects after several months as in the case of 
doxorubicin and bleomycin which induces cardiomyopathy and pulmonary fibrosis. To avoid these complications, measures have been implemented to limit the dosage of drugs over time. Studies report that patients are susceptible to have chronic health conditions several years after diagnosis.

\subsection{Combination therapy}

Administration of a single anticancer drug is not a promising option for the effective treatment of cancer. Tumors exhibit different characteristics from one type to another. To overcome these issues, anticancer drugs were combined and have been practiced in the clinic widely to treat testicular teratomas and high-grade lymphomas. Combination therapy significantly improves the rate of cure in comparison to single-agent therapy. It is always preferable to combine drugs of different classes to effectively improve activity against the resistant cells and to reduce the growth of new resistant subclones. It is also important to consider the toxicity profiles of the drugs before using them for combination therapy.

\section{Importance of nanomaterials in cancer treatment}

Among the treatment methods used against fighting cancer, chemotherapy is the most applied one in the clinical setting. Since it is a systemic treatment irrespective of the route of administration it causes irreversible side effects to the patients as discussed previously.

Therefore it is crucial to explore strategies to target these cytotoxic drugs into the tumor site so that the toxic effects can be minimized. Researchers began to utilize the wide potential of nanomaterials to circumvent the issues with conventional chemotherapeutic agents.

To tackle the issues with the conventional chemotherapeutic agents, novel nano-drug carriers were developed. These include polymeric nanoparticles, micelles, liposomes, magnetic nanoparticles, mesoporous silica nanoparticles, metal nanoparticles, and carbon-based nanosystems (carbon nanotubes, carbon dots, and graphene oxide). ${ }^{39}$

With increasing advances in the field of nanomaterials chemistry, researchers were able to synthesize nanoparticles with tunable physicochemical properties. These nanocarriers were combined with biologically active molecules (BAM) such as proteins, genes, nucleotides, and enzymes to fabricate efficient, safe, and targeted nano-drug delivery systems. Any drug delivery system (DDS) be it magnetic nanoparticles or polymeric nanoparticles should comply with the following criteria. ${ }^{40}$

(1) It should be inert once administered and should not cause any adverse reactions. It should also be able to escape from the RES (reticuloendothelial system) uptake.

(2) The DDS should be able to transport and protect the BAM (e.g., hydrolysis or enzymatic degradation) until it reaches the desired site.

(3) DDS has to avoid burst release of the drugs and must be able to release the drugs in a controlled fashion in the desired site or in its vicinity.
(4) The DDS must be able to get eliminated from the system after delivering the drug cargo.

There are several methods to fabricate the drug delivery systems with these requirements and few of them are common to all and a few are specifically crafted to meet the need of the drug carrier's architecture and structure. Some drug delivery systems are suitable for conjugating the drugs (e.g., polymerdrug conjugates) in a covalent manner and some by noncovalent approaches (e.g., magnetic nanoclusters).

\subsection{Nanomaterials as drug carriers}

Drug carriers that are based on nanomaterials have several advantages in comparison with free drugs. Nano drug carriers can significantly improve the solubility of the drugs, avoid premature release, and can increase drug stability and bioavailability. Nanomaterial based drug carriers are presented in Fig. 2 .

Nano drug carriers are principally designed to improve the selectivity to effectively target the drugs to the desired site and to reduce the immunogenicity by either coating the surface with biocompatible materials or by chemically functionalizing the surface with antibodies, peptides, cell-membrane, tunable surfactants, and natural polysaccharides. ${ }^{41-43}$ These ligandmodified nanocarriers not only improve the biocompatibility but also help the drug carriers to cross certain barriers such as the cerebrospinal fluid barrier or blood-brain barrier. Ligandmodified nanocarriers possess the ability to pass through the cell membrane and deliver the drugs in a particular environment.

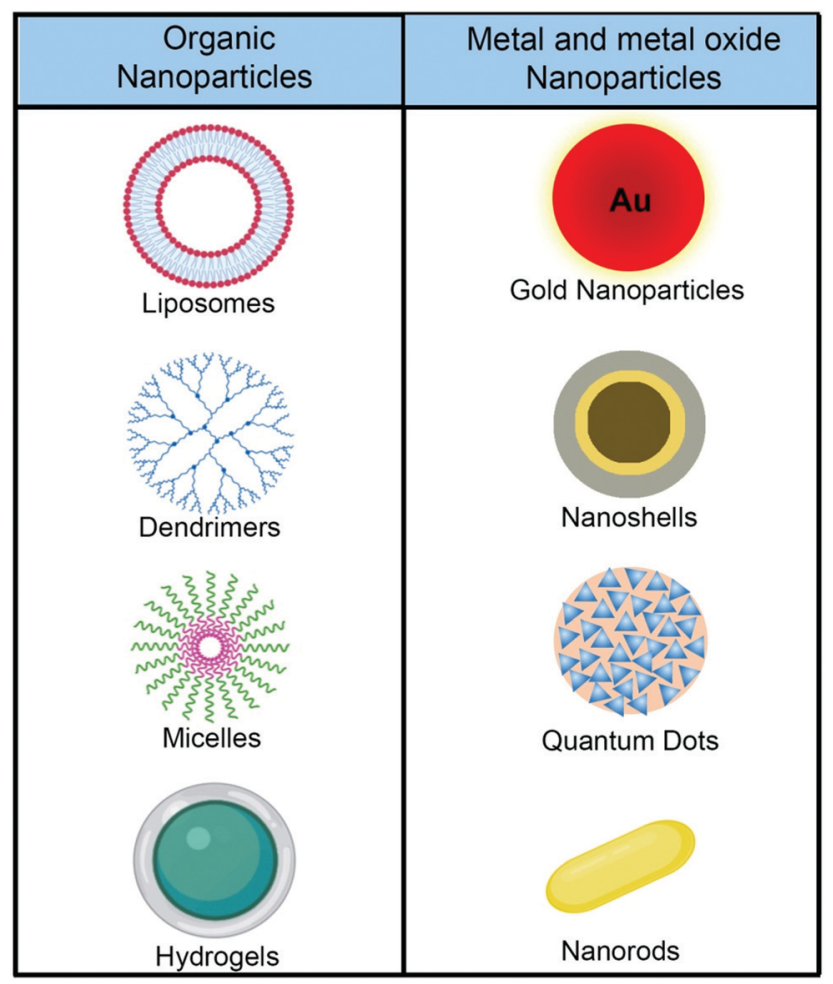

Fig. 2 Nanomaterial based drug carriers. 
One example to demonstrate the beneficial effects of nanodrug carrier and ligand conjugation is Hyaluronic acid. This ligand conjugation has shown promising results in boosting the anti-tumor action against breast cancer cells, melanoma stem-like cells, and pulmonary adenocarcinoma cells. Ligandappended nano-drug delivery systems are beneficial but their construction is labor-intensive and requires several targeting designs which have to include the physiological variables of disease status, blood flow, and tissue architecture. ${ }^{44,45}$

Stimuli-responsive nanocarriers have demonstrated the ability to release the drugs in a controlled manner by using external stimuli such as heat, light, ultrasound, $\mathrm{pH}$, and ionic strength which can increase the targeting ability of the nanocarrier along with an increased accumulation of drugs at the required site. ${ }^{9,46,47}$

Polymers derived from both natural and synthetic origin such as poly(lactic acid) (PLA), poly(lactide-co-glycolide) (PLGA), polycaprolactone (PCL), chitosan, and collagen are extensively used for biomedical applications. The imaging and therapeutic moieties can be encapsulated into the polymers with or without surface modification. Drugs encapsulated in the polymers can be released in a sustained manner via polymer degradation under physiological conditions, diffusion of the drugs through the matrices present in the polymer, or changes in the conjugation due to cell environment stimuli such as $\mathrm{pH}^{48}$ Several hollow and porous based nanostructures have been developed using silica, manganese, and gold to function as drug carriers. ${ }^{49,50}$

\subsection{Targeting drugs to tumor microenvironment}

The development of new therapies to combat against cancer essentially needs a detailed understanding of the tumor microenvironment. Newer therapies and drugs are developed by understanding the differences between the normal and the cancer tissues which includes $\mathrm{pH}$, vascular abnormalities, metabolic states, perfusion, and oxygenation. There are two modes of drug targeting to the tumor microenvironment passive targeting and active targeting (Fig. 3).

\subsection{Passive targeting}

The vascular pathophysiology of the tumor microenvironment is characterized by leaky vasculatures and structural irregularities. These structural irregularities help the nanocarriers to deliver the drug cargo into the tumor microenvironment through convection or passive diffusion. ${ }^{51}$ Large molecules are transported through convection via large pores when the rate of net filtration is at zero and low molecular weight compounds are transported through diffusion. Convection refers to the movement of molecules within the fluids and diffusion is the process in which molecules are transported across the cell membrane. ${ }^{52}$

Diffusion is the major mode of drug transport as transport through convection is poor because of interstitial hypertension. The nanocarriers are selectively then accumulated in the tumour by the Enhanced Permeability and Retention effect (EPR effect). EPR has become an essential standard in designing tumor-targeted drugs. EPR is used as a guiding principle to deliver the drugs in the tumor through the nanocarriers and almost applicable to all tumors occurring in humans only with the exception of hypovascular tumors which include prostate and pancreatic cancer. ${ }^{53}$

EPR effect can be an optimal way to deliver the drugs if the nanocarrier can bypass the immune surveillance with longer circulation times. Hence through EPR high concentrations of drugs can be delivered at the site of the tumour. ${ }^{54}$ To achieve this, the nanocarrier has to possess the following three important properties.

(i) Size. The size of the nanocarrier has to be less than $400 \mathrm{~nm}$ in order to be efficiently extravasated from the leaky vasculature. And the minimum size of the nanocarrier should

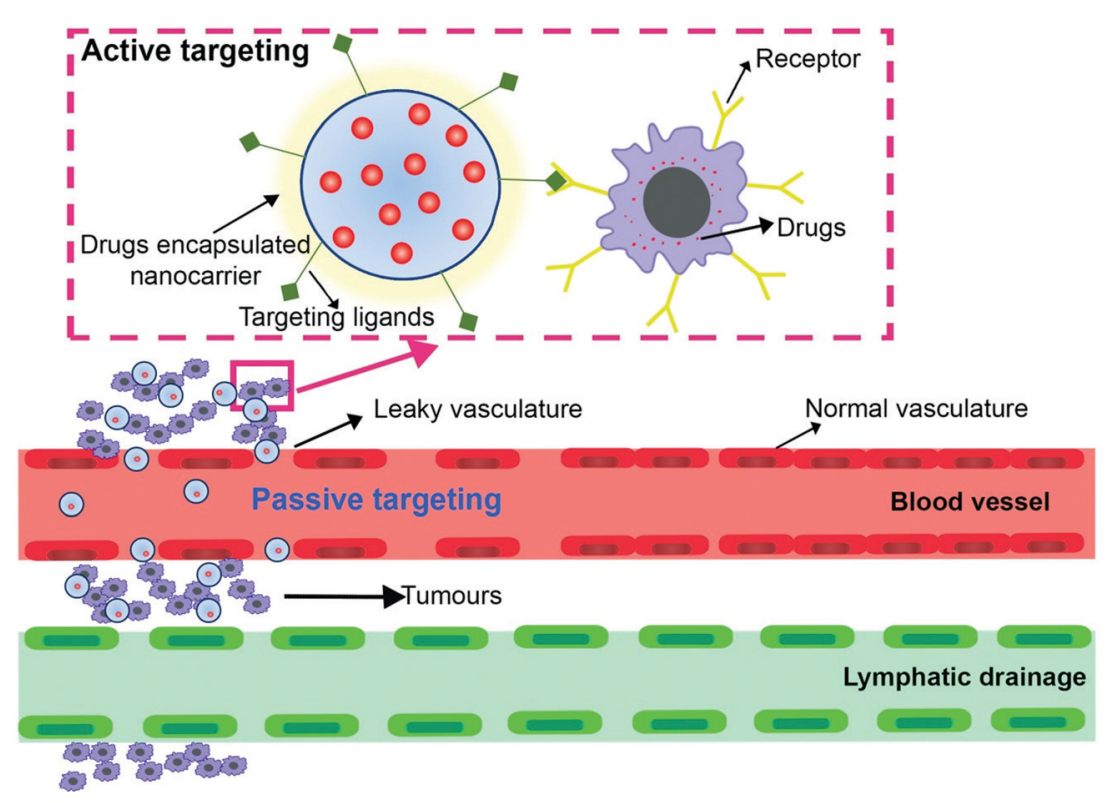

Fig. 3 Mechanism of active and passive drug targeting by nanodrug carriers. 
be greater than $10 \mathrm{~nm}$ so as to avoid being filtered by the kidneys and lesser than $100 \mathrm{~nm}$ to avoid the specific capture by the liver. Hence the ideal size of the nanocarrier should be between 10 and $100 \mathrm{~nm}$.

(ii) Charge. The charge of the nanocarrier has to be either anionic or neutral for efficient evasion of the renal elimination.

(iii) Escape from RES. The nanocarrier has to be biocompatible enough to escape the reticuloendothelial system avoiding its destruction by phagocytosis through opsonization.

Passive targeting is highly dependent on the degree of the presence of leaky vasculatures. Thus, the degree of extravasation may vary with each specific tumour type. And the presence of high interstitial fluid pressure in solid tumors avoids the uptake and distribution of drugs in the tumor site which can be successfully overcome by using nanocarriers (Table 1).

\subsection{Active drug targeting}

In active drug targeting, the surface of the nanocarrier is conjugated with ligands that have an affinity to bind with the receptors present on the surface of the cancer cells. The ligand is carefully chosen to specifically bind to the receptors that are overexpressed on the surface of the cancer cells and not in normal cells. Commonly overexpressed receptors are transferrin receptors, glycoproteins, folate receptors, and epidermal growth factor receptors (EGFRs). ${ }^{55-57}$

A ligand-specific to the overexpressed receptor is conjugated to the surface of the nanocarrier and administered into the system. The ligand present on the nanocarrier then binds to the receptor and thus delivers the cargo into the tumour. Monoclonal antibodies (mAbs), antibody fragments, or non-antibody ligands are chosen as targeting ligands. The effectiveness of targeting the nanocarriers greatly depend on the binding affinity. ${ }^{58,59}$

Active targeting is achieved at different levels based on the penetration at the surface of the cell, organ, or subcellular levels. It is to be noted that in cases of solid tumour active targeting begins with the drugs passively accumulating in the tumour tissue. Hence, it is of crucial importance that the drug carriers must also satisfy the primary requirements stated for the passive targeting systems.

Active targeting drug systems are more selective than the passive targeting systems because of their selectivity which is specifically effective in cases of solid tumours, leukaemia and other tumour cells that are present in the blood circulation. Owing to their specific nature they are more effective in treating the diseases in comparison with the passive targeting systems.
To design effective active targeting drug system detailed information about the surface receptors (lipoproteins, proteins and glycoproteins) that are abundantly present on the cancer cell surface is required. It is also important to identify ligands such as lectins, saccharides, antibodies, antibody fragments, enzymes, or enzyme inhibitors, and peptides that have a strong affinity to bind to the cell surface receptors. Conjugation of these ligands to the drug carrier ensures the safe delivery of the payload specifically to the desired site thereby avoiding the non-specific accumulation of drugs in the healthy tissues.

\section{Nanotheranostics}

Theranostics is an emerging field of research and most importantly an essential keyword that is often found in the cancer research. ${ }^{60}$ The concept of theranostics is based on the ability of a therapeutic agent to act as a drug as well as a diagnostic agent. It has a wide impact on strategizing the treatment plan for the patients based on the molecular information that is obtained from molecular imaging and thus helps to optimize the drug dosage. Theranostics aims to improve the overall healthcare system and to reduce the cost of the treatments by monitoring the response to the treatment, increasing the drug safety and efficacy and eliminating the treatments that are not necessary. Therefore the emerging field of theranostics holds promise to provide a great opportunity to the diagnostics and pharmaceutical companies to meet the financial and regulatory constraints outlined by the Food and Drug Administration (FDA).

In the field of cancer research, the idea of theranostics is aimed at reducing the burden of cancer-based on the assumption that if cancer growth can be hampered at the early stage during the molecular diagnosis, it will subsequently help the cancer treatment by retarding the growth of the tumor. The early stage of theranostics witnessed the addition of an imaging probe to the drug carrier loaded with the therapeutic agent. The imaging probe is a contrast agent that can be detected by either one of the imaging modalities such as magnetic resonance imaging (MRI), computed tomography (CT) and positron emission tomography (PET).

Cancer research has undergone a lot of changes over the past few years and no longer viewed only from the point of reducing tumour growth. Cancer cells communicate with a complex mixture of cells present in the surroundings such as endothelial cells, fibroblasts, neovasculature, and immune cells. Cancer cells also develop various survival strategies that are considered as the six hallmarks of cancer. ${ }^{61}$ They are

Table 1 Passively targeted FDA approved nanoparticle formulations

\begin{tabular}{|c|c|c|c|}
\hline Nanocarrier & Biomedical payload & Commercial name & Indications \\
\hline \multirow[t]{2}{*}{ Nanoparticles } & Albumin-paclitaxel & Abraxane & Metastatic breast cancer \\
\hline & Doxorubicin & Transdrug & Hepatocarcinoma \\
\hline \multirow[t]{4}{*}{ Liposomes } & Doxorubicin & Doxil & Metastatic breast cancer, ovarian cancer and kaposis sarcoma \\
\hline & Vincristine & Onco-TCS & Non-Hodgkin lymphoma \\
\hline & Doxorubicin & Myocet & Breast cancer \\
\hline & Daunorubicin & DaunoXome & Kaposis sarcoma \\
\hline
\end{tabular}


sustained angiogenesis, unlimited replication, insensitivity to anti-growth signals, evasion of apoptosis, self-sufficiency in growth signals and metastasis. Continued exploration about the cancer mechanism added two more additional hallmarks i.e., escape from the immune destruction and deregulation of cellular energetics. ${ }^{62}$

Understanding the mechanisms of tumour growth is a necessary factor to develop therapeutics to treat and target the drugs to the cancer site. These agents are designed to target and attack the factors that are responsible for promoting the growth of the tumour. These targets are generally associated with genetic aberrations such as deletions, translocations (e.g., gene fusion), amplifications, and mutations (single-base substitutions). For example, the ErbB2 (amplification) is the target oncogene that governs the oncogenic potential of tumour cells in breast cancer. In certain cases, cancer cells can also become dependent on non-oncogenes, which contributes to the proliferation and survival of the cells under stress condition.

Oncogenes often undergo mutations at the same amino acid positions which increases the activity of the corresponding proteins. For example, in non-small-cell lung cancer, the mutant L858R EGFR is a mutated form of the epithelial growth factor receptor (EGFR). This mutant can be imaged using CT or PET techniques, immunohistochemical analysis and fluorescence in situ hybridization analysis. ${ }^{63-65}$

In the case of multiple tumour lesions, patients are subjected to repetitive biopsy procedures which causes severe trauma. In this context, non-invasive imaging techniques are beneficial and superior over the invasive biopsy procedures and thus can reduce the trauma in the patients.

As discussed earlier, cancer cells develop high resistance and even with high doses of chemotherapy and extensive radiotherapy, it is very difficult to completely eradicate the growth of the tumor. And in most cases, the tumour that acquires resistance to the previous treatment methods starts to recur because of the ability of the cancer cells to adapt and survive. This shows that the tumour can be effectively treated by combining a therapeutic agent and an imaging agent into a single platform.

Several nanomaterials have been designed to address the existing issues with conventional treatment options. The term nanomedicines coined by the National Institutes of Health (NIH) denotes nanoparticle-based materials that are composed of inorganic or organic nanomaterials with therapeutic and imaging agents that can diagnose, monitor and control the biological systems. Nanomaterials can be utilized to load multiple types of anti-cancer drugs and in certain cases, the nanomaterial itself possess inherent anticancer and imaging properties. ${ }^{66}$

These nanomaterials can target the surface receptors present in the tumour cells and can also be loaded with multimodal imaging agents. Theranostic nanoparticles help to monitor the biological response to the treatment given and thus helps to increase the efficacy and safety of the drug. ${ }^{67}$ Several new advances have been reported in the field of theranostic nanoparticles which include the MRI contrast using superparamagnetic nanoparticles, ${ }^{68}$ Carbon nanomaterials for photoacoustic imaging and optical imaging, ${ }^{69}$ stealth and photothermal property of polymer-based nanoparticles, ${ }^{70}$ nanoparticle-based gene delivery vehicles, ${ }^{71}$ multifunctional nanoparticles, ${ }^{72}$ and stimuli-responsive nanomaterials. ${ }^{73}$

\subsection{Design of theranostic nanoparticles}

In general, theranostic nanoparticles have the following three components - a nanocarrier, biomedical payload and a linker/ surface modifier depending on the role of the nano-drug carrier and its targeted site (Fig. 4). It is essential that the nano-drug carrier should be able to protect the payload and deliver it to the required site. The surface of the nano-drug carriers is modified with linkers to conjugate receptors or imaging moieties. Modification of the surface imparts the theranostic ability to the carrier and also makes them highly biocompatible, improves the circulation time, and provides specific target binding ability. The essential components for the fabrication of the theranostic nanoparticles are briefly listed in Table 2 .

\subsection{Imaging payloads}

Various imaging modalities along with their characteristics are represented in Table 3.

(i) Optical imaging. Optical based molecular imaging is primarily based on the use of organic fluorophores such as cyanine 5.5 (Cy5.5) and fluorescein isothiocyanate (FITC) to monitor the molecular changes that are happening in the biological systems. The visible light source used to excite the organic fluorophores doesn't cause any harmful effect to the tissue and is specifically confined to the cells. Organic fluorophores/dyes are prone to photobleaching effects and are also toxic. To overcome the issues of toxicity and photostability, several methods have been opted to encapsulate the organic dyes in biocompatible matrices such as silica ${ }^{74}$ and natural/ synthetic polymers. ${ }^{75}$ This encapsulation enables the protection of the organic dye from premature release which will significantly impact the signal output. In this context, twophoton dyes that are excitable by the NIR light was developed $^{76}$ as NIR light absorption by the skin is very minimal.

Several theranostic nanoparticles were synthesized with applications in cancer. Chitosan-based theranostic nanoparticles were prepared by Park and Kwon et al. ${ }^{77}$ in this study

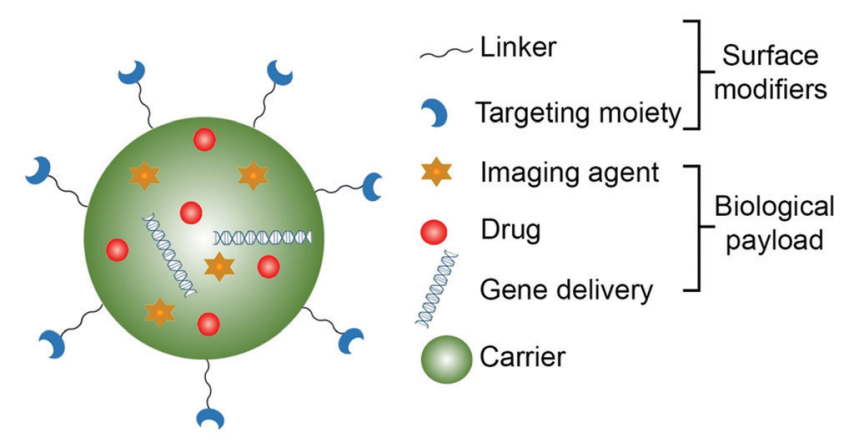

Fig. 4 Schematic illustrations of a theranostic nanoplatforms. 
Table 2 Components of theranostic nanoparticles

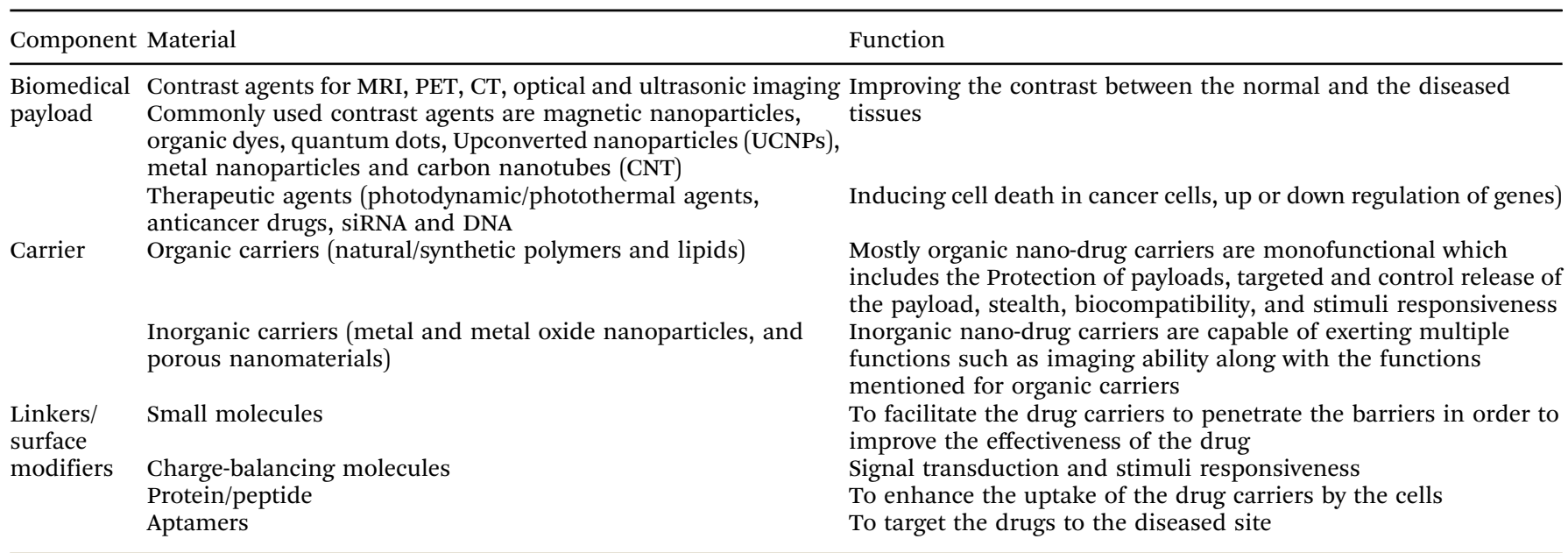

Table 3 Characteristics of Imaging modalities

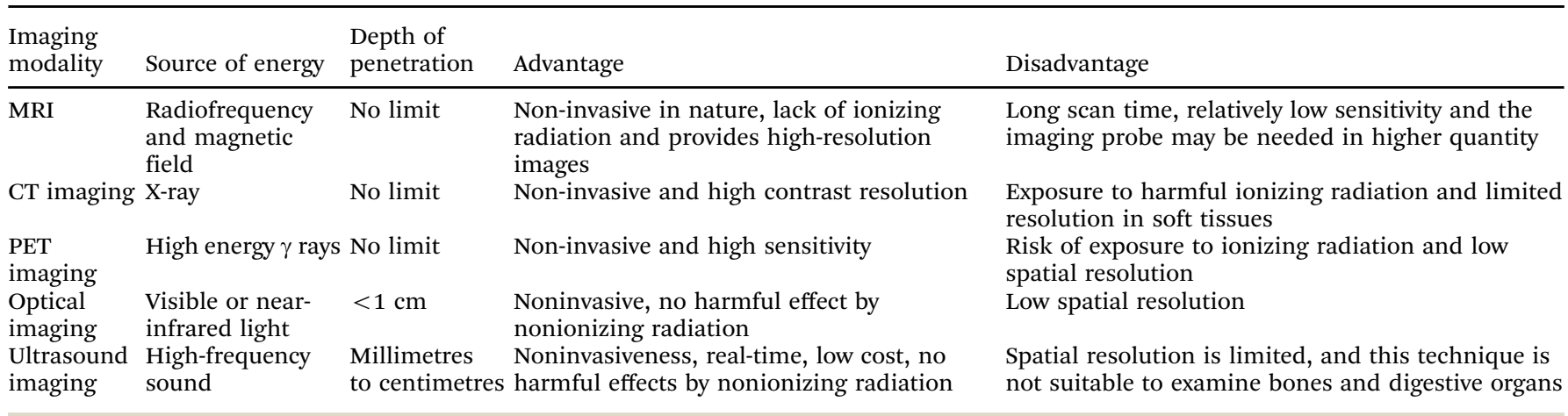

the nanoparticles were loaded with the drug paclitaxel (PTX) and a NIRF dye Cy5.5. Several strategies were opted to synthesize theranostic nanoparticles based on NIRF dyes for imaging and an anticancer drug for therapy. These theranostic nanoparticles were also conjugated with targeting moieties to target them to the required site. ${ }^{78-80}$

Upconversion nanoparticles (UCNPs) are made up of inorganic materials that can absorb light in NIR range and emit light in the UV or visible range. UCNPs are comparable to two-photon dyes and are used to monitor important cell events even though they are larger than the organic dyes. ${ }^{81}$ Organic dyes can also be linked to fluorescence quenching moieties such as gold nanoparticle via peptide linkers. Delivering this particle in the environment with peptide cleaving enzymes, the fluorescence of the organic dyes is restored as the fluorescence resonance energy transfer (FRET) mechanism is absent. Thus the restored fluorescence signal can be used to monitor the presence of certain enzymes in a biological system. ${ }^{82}$

(ii) Magnetic resonance imaging. Magnetic nanoparticles, composed of oxides of iron and manganese, rare earth metal oxides and alloy systems are the most studied materials for biomedical applications such as magnetic separation, stem cell trafficking and molecular imaging. ${ }^{83}$ Magnetic nanoparticles have the potential to act as $T_{1}$ and $T_{2}$ contrast agents in MRI.
Design of core-shell nanostructures has enabled the combination of the imaging probe with the drug carrier. Several researchers have combined metal and organic frameworks to design theranostic nanoparticles for loading the drugs and imaging probes and have successfully demonstrated the same in in vivo models. $^{84,85}$

Several intriguing works have also been reported such as the utilization of the core-shell structure of $\mathrm{MnO} @ \mathrm{Mn}_{3} \mathrm{O}_{4}$ as a drug carrier as well as an imaging probe. The $\mathrm{Mn}_{3} \mathrm{O}_{4}$ acts as a hollow container to load the anticancer drug and the $\mathrm{MnO}$ is specific to tumour environment which gets leached and releases $\mathrm{Mn}$ (II) ions thus allowing to map the low $\mathrm{pH}$ sites through MR imaging. ${ }^{86-88}$ The leaching of $\mathrm{MnO}$ in the tumour cells can also improve the tumouricidal efficacy of anticancer drugs such as doxorubicin. ${ }^{89}$ In yet another study based on the leaching of platinum (Pt) from FePt, the leaching effect of the metal has been found to increase the killing of cancer cells. ${ }^{90,91}$

Several polymer-based theranostic nanoparticles with the potential to act as MR probes have been fabricated by several groups. In a study by Kataoka et al. core-shell micelles were prepared and loaded with the therapeutic agent 1,2-diaminocyclohexane-Pt(II) and imaging agent Gd-DTPA. ${ }^{92}$ The therapeutic payload of these theranostic nanoparticles also includes photosensitizer dyes for photodynamic therapy (PDT). ${ }^{93,94}$ 
(iii) PET imaging. Along with the drugs, radiotracers for PET imaging are also conjugated to the theranostic nanoparticles. PET images help to understand the pharmacokinetic behaviour and accumulation of nanoparticles in the system. ${ }^{95}$ Researchers have also combined the PET probes with other contrast agents together in a single system to function as a multimodal imaging agent which improves the diagnostic accuracy. ${ }^{96,97}$

(iv) Other imaging payloads. Aptamer-conjugated targeted theranostic nanoparticles were prepared by Yeh et al. which can effectively target human leukemic CCRF-CEM cells and release the drugs by application of ultrasound to a specific region. This aptamer conjugated system is advantageous as it can help to prevent the premature release of drugs. ${ }^{98}$ Metallic nanoparticles such as gold ( $\mathrm{Au})$, silver $(\mathrm{Ag})$ and palladium $(\mathrm{Pd})$ have been synthesized and investigated for various biomedical applications. Au nanoparticles can be tuned to absorb NIR light which can be converted into heat to kill the cancer cells via hyperthermia. ${ }^{99}$ Carbon nanomaterials such as carbon nanotubes, graphene oxide and carbon nanodots are also attractive for their tunable photoluminescent properties and have been extensively used for PDT, photothermal therapy, ${ }^{100}$ photoacoustic imaging, ${ }^{101}$ and optical imaging. ${ }^{102}$

\subsection{Therapeutic agents}

Chemotherapeutic drugs interfere with the DNA function and synthesis and thus inhibit the growth and spreading of tumour. Commonly used chemotherapeutic drugs are methotrexate (MTX), 6-mercaptopurine (6-MP), hydroxyurea (hydroxycarbamide), 5-fluorouracil (5-FU), doxorubicin (DOX), daunorubicin and etoposide. Side effects caused by chemotherapy and the use of nanocarriers in overcoming the side effects have been discussed in Section 1.6.1.

Several other strategies were developed for the treatment of cancer and they have shown promising results. Targeted antibody therapy is based on employing monoclonal antibodies (mAbs) and targeting agents such as tyrosine kinase inhibitors (TKI). ${ }^{103}$ Selected gene sequences were delivered into the specific cells of patients to repair the defects in the gene that occurred because of the mutation. ${ }^{104}$ This technique is known as gene therapy and has been tested against different types of cancer in clinical settings. Presence of upregulated or inappropriately expressed genes cause uncontrolled cell growth in cancer cells. Identification of these target genes can lead to the development of selective anticancer agents and lessen the side effects of chemotherapy.

Hyperthermia-based cancer treatments have also received much attention as an alternative to existing cancer therapies. In hyperthermia, the tumour region is exposed to temperatures (41-45 ${ }^{\circ} \mathrm{C}$ ) to induce cellular changes by damaging intracellular proteins. ${ }^{105}$ Gold and magnetic nanoparticles have been used extensively as hyperthermia inducing agents. ${ }^{106}$

Another alternative nanoparticle-based non-invasive strategy is photodynamic therapy (PDT). In PDT, photosensitizer molecules are conjugated to the nanoparticles and are excited at specific wavelengths to generate reactive oxygen species
(ROS). Excess generation of ROS kills cancer cells by damaging nucleic acids and proteins. ${ }^{107}$

\subsection{Surface modifiers}

Generally, tumor blood vessels are presented with fenestrations and they have the potential to form new blood vessels to provide the tumors with the supply of oxygen and nutrients. ${ }^{108,109}$ These abnormalities present in the tumor blood vessels are utilized in delivering drugs to the tumor microenvironment via EPR effects. Hence, nanoparticles should be hemocompatible and should not be captured by the RES. It is important that the nanoparticles are taken up by the cells in the target site before releasing their payload. These requisites necessitate the need for research in surface modifications of the nanoparticles. As discussed earlier, the most commonly used nano-based drug carriers are liposomes, surface-modified nanomaterials, natural polymers (e.g., cellulose, dextran, starch, and hyaluronic acid), and synthetic polymers. ${ }^{110,111}$

To improve the therapeutic efficacy and the physicochemical properties of the nanoparticles surface modification is essential. Polyethylene glycol (PEG) is the most commonly used material in the surface engineering of nanoparticles due to its hydrophilic, non-antigenic, and non-immunogenic nature. PEG can thus act as a steric barrier and prevent the nanoparticle from opsonization, macrophage uptake, and avoids degradation by the immune system. Thus the coating of PEG over the nanoparticles improves the circulation time of the nanoparticles. ${ }^{112}$

Silica nanoparticles are also widely used to render solubility and biocompatibility to inorganic nanoparticles. The surface of the silica nanoparticles can be easily modified with various moieties such as antibodies, DNA, lectins, proteins, oligonucleotides, and oligopeptides. Overexpressed receptors on the surface of cancer cells can act as targets. Nanoparticles are mostly conjugated with aptamers, antibodies and their fragments, ligand receptors such as carbohydrates, peptides, and vitamins, and macromolecules that have a high binding affinity with the target receptors.

Conjugation of the ligand moieties depends on the type of functional group present on the surface of the nanoparticles. The binding efficiency of antibody-based targeting systems has been demonstrated in clinical settings and several antibodies have been approved by the FDA. Few examples of FDA-approved antibodies available in the market are Trastuzumab (Herceptin) ${ }^{113}$ and Bevacizumab (Avastin). ${ }^{114}$

Aptamers have the affinity to bind to several targets such as carbohydrates, soluble proteins, transmembrane proteins, intracellular proteins, and drugs. ${ }^{115}$ The low extracellular $\mathrm{pH}$ of the cancer cells can also be used as a stimulus to activate the release of drugs specifically in the tumor environment. Further, nanoparticles with positive surface charge can actively bind to the surface of the cancer cells through electrostatic interactions as the cell membrane is negatively charged. ${ }^{70}$ Nanoparticles, surface modified with amino acid, mannitol, and glucose can help the nanoparticles to deliver the drugs bypassing the blood-brain barrier (BBB). ${ }^{116,117}$ These surface modifications 
allow the theranostic agents to act efficiently in molecular diagnosis and therapy.

\section{Conclusion}

In Conclusion, the impact of molecular imaging with the advances in MRI has proved to be effective in achieving an early diagnosis. Although contrast agents used traditionally in diagnosing diseases are efficient, they suffer from a variety of adverse effects that may be fatal. The superior properties of magnetic nanomaterials have been rescuing these side effects due to their remarkable properties, specifically the ability to alter the $T_{1}$ and $T_{2}$ properties of the surrounding tissues generating contrast differences in MRI. On the other hand, several chemotherapeutic agents and radiotherapy used for treating cancers experience side effects due to their improper distribution and non-targeted radiation therapy. To relieve these failures of conventional cancer treatment modalities, nanomaterials prove to be effective as a nanocarrier and as a targeted drug delivery system whereby the efficiency of the chemotherapeutic drug or agent is utilized to the fullest at the target cancer site. Functionalization or conjugation of targeting moieties such as folic acid allows for active targeting while passive targeting is achieved due to the effective size being good enough to traverse through the leaky microvasculature in cancer tissues. Nanotheranostics have recently gained importance due to the proficiency offered by various nanomaterials to generate diagnostic abilities in various imaging modalities along with therapeutic action on cancer cells. These nanotheranostic agents are used either as a multifunctional nanoformulation or a nanomaterial with surface modifications that have the efficiency expected for theragnosis. Hence, nanomaterials prove to be highly efficient in generating theragnosis overcoming the deficiencies noted with conventional diagnostic and therapeutic agents used for cancers.

\section{Conflicts of interest}

There are no conflicts to declare.

\section{References}

1 H.-C. Huang, S. Barua, G. Sharma, S. K. Dey and K. Rege, J. Controlled Release, 2011, 155, 344-357.

2 Z. Liu, F. Kiessling and J. Gätjens, J. Nanomater., 2010, 2010, 51.

3 G. Amsaveni, A. S. Farook, V. Haribabu, R. Murugesan and A. Girigoswami, Adv. Sci., Eng. Med., 2013, 5, 1340-1348.

4 B. Das, A. Girigoswami, P. Pal and S. Dhara, Mater. Sci. Eng., C, 2019, 102, 427-436.

5 S. Yamini, M. Gunaseelan, G. Kumar, S. Singh, G. C. Dannangoda, K. S. Martirosyan, D. K. Sardar, S. Sivakumar, A. Girigoswami and J. Senthilselvan, Microchim. Acta, 2020, 187, 1-10.
6 M. L. James and S. S. Gambhir, Physiol. Rev., 2012, 92, 897-965.

7 F.-F. Teng, X. Meng, X.-D. Sun and J.-M. Yu, Int. J. Nanomed., 2013, 8, 3703.

8 B. Das, A. Girigoswami, A. Dutta, P. Pal, J. Dutta, P. Dadhich, P. K. Srivas and S. Dhara, ACS Biomater. Sci. Eng., 2019, 5, 3549-3560.

9 V. Haribabu, P. Sharmiladevi, N. Akhtar, A. S. Farook, K. Girigoswami and A. Girigoswami, Curr. Drug Delivery, 2019, 16, 233-241.

10 V. Haribabu, K. Girigoswami, P. Sharmiladevi and A. Girigoswami, ACS Biomater. Sci. Eng., 2020, 6, 4377-4389.

11 B. R. Smith, P. Kempen, D. Bouley, A. Xu, Z. Liu, N. Melosh, H. Dai, R. Sinclair and S. S. Gambhir, Nano Lett., 2012, 12, 3369-3377.

12 A. V. Annapragada, E. Hoffman, A. Divekar, E. Karathanasis and K. B. Ghaghada, Methodist DeBakey cardiovascular journal, 2012, 8, 18-22.

13 B. R. Smith, Z. Cheng, A. De, A. L. Koh, R. Sinclair and S. S. Gambhir, Nano Lett., 2008, 8, 2599-2606.

14 X. Duan and Y. Li, Small, 2013, 9, 1521-1532.

15 R. M. Ferguson, A. P. Khandhar, H. Arami, L. Hua, O. Hovorka and K. M. Krishnan, Biomed. Tech., 2013, 58, 493-507.

16 B. Polyak and B. Cordovez, Nanomedicine, 2016, 11(3), 189-192.

17 K. Mohr, M. Sommer, G. Baier, S. Schöttler, P. Okwieka, S. Tenzer, K. Landfester, V. Mailänder, M. Schmidt and R. G. Meyer, J. Nanomed. Nanotechnol., 2014, 5, 1-10.

18 G. Mistlberger and I. Klimant, Bioanalytical Rev., 2010, 2, 61-101.

19 H. S. Choi, W. Liu, P. Misra, E. Tanaka, J. P. Zimmer, B. I. Ipe, M. G. Bawendi and J. V. Frangioni, Nat. Biotechnol., 2007, 25, 1165.

20 R. A. Cairns, I. S. Harris and T. W. Mak, Nat. Rev. Cancer, 2011, 11, 85.

21 J.-Q. Chen and J. Russo, Biochim. Biophys. Acta, Rev. Cancer, 2012, 1826, 370-384.

22 R. J. Gillies and D. L. Morse, Annu. Rev. Biomed. Eng., 2005, 7, 287-326.

23 H. C. Le, M. Lupu, K. Kotedia, N. Rosen, D. Solit and J. A. Koutcher, Magn. Reson. Med., 2009, 62, 1112-1119.

24 I. Park, P. E. Larson, M. L. Zierhut, S. Hu, R. Bok, T. Ozawa, J. Kurhanewicz, D. B. Vigneron, S. R. VandenBerg and C. D. James, Neuro-Oncology, 2010, 12, 133-144.

25 F. A. Gallagher, M. I. Kettunen, S. E. Day, D.-E. Hu, J. H. Ardenkjær-Larsen, P. R. Jensen, M. Karlsson, K. Golman, M. H. Lerche and K. M. Brindle, Nature, 2008, 453, 940.

26 K. Ward, A. Aletras and R. S. Balaban, J. Magn. Reson., 2000, 143, 79-87.

27 O. Togao, T. Yoshiura, J. Keupp, A. Hiwatashi, K. Yamashita, K. Kikuchi, Y. Suzuki, S. O. Suzuki, T. Iwaki and N. Hata, Neuro-Oncology, 2013, 16, 441-448.

28 A. Y. Sheu, Z. Zhang, R. A. Omary and A. C. Larson, Invest. Radiol., 2013, 48, 492. 
29 P. Sharmiladevi, V. Haribabu, K. Girigoswami, A. S. Farook and A. Girigoswami, Sci. Rep., 2017, 7, 11179.

30 N. Lee, Y. Choi, Y. Lee, M. Park, W. K. Moon, S. H. Choi and T. Hyeon, Nano Lett., 2012, 12, 3127-3131.

31 M. H. Pablico-Lansigan, S. F. Situ and A. C. S. Samia, Nanoscale, 2013, 5, 4040-4055.

32 R. Baskar, K. A. Lee, R. Yeo and K.-W. Yeoh, Int. J. Med. Sci., 2012, 9, 193.

33 A. P. Brown, J. Chen, Y. J. Hitchcock, A. Szabo, D. C. Shrieve and J. D. Tward, J. Clin. Endocrinol. Metab., 2008, 93, 504-515.

34 M. Dollinger and E. H. Rosenbaum, Everyone's Guide to Cancer Therapy;: How Cancer Is Diagnosed, Treated, and Managed Day to Day, Andrews McMeel Publishing, 2002.

35 F. J. Berkey, Am. Fam. Physician, 2010, 82, 381-388.

36 Cancer: Principles and Practice of Oncology, ed. V. T. Devita, S. Hellman and S. A. Rosenberg, Lippincott Williams \& Wilkins Publishers, 6th edn, 2001.

37 R. K. Jain, Cancer Res., 1987, 47, 3039-3051.

38 P. G. Corrie, Medicine, 2008, 36, 24-28.

39 R. Wang, P. S. Billone and W. M. Mullett, J. Nanomater., 2013, 2013, 1.

40 P. V. Devarajan and S. Jain, Targeted drug delivery: concepts and design, Springer, 2015.

41 A. Jin, Y. Wang, K. Lin and L. Jiang, Bioact. Mater., 2020, 5, 522-541.

42 L. Huang, J. Huang, J. Huang, H. Xue, Z. Liang, J. Wu and C. Chen, Biomater. Sci., 2020, 8, 2376-2393.

43 S. Y. Fam, C. F. Chee, C. Y. Yong, K. L. Ho, A. R. Mariatulqabtiah and W. S. Tan, Nanomaterials, 2020, 10, 787.

44 S. Muro, J. Controlled Release, 2012, 164, 125-137.

45 N. Wathoni, A. N. Nguyen, A. Rusdin, A. K. Umar, A. F. A. Mohammed, K. Motoyama, I. M. Joni and M. Muchtaridi, Drug Des. Dev. Ther., 2020, 14, 4387.

46 P. Zhang, D. Gao, K. An, Q. Shen, C. Wang, Y. Zhang, X. Pan,

X. Chen, Y. Lyv and C. Cui, Nat. Chem., 2020, 12, 381-390.

47 P. Mi, Theranostics, 2020, 10, 4557.

48 A. Z. Wilczewska, K. Niemirowicz, K. H. Markiewicz and H. Car, Pharmacol. Rep., 2012, 64, 1020-1037.

49 J. L. Vivero-Escoto, I. I. Slowing, C.-W. Wu and V. S.-Y. Lin, J. Am. Chem. Soc., 2009, 131, 3462-3463.

50 K. Möller and T. Bein, Chem. Mater., 2019, 31, 4364-4378. 51 V. P. Torchilin, Eur. J. Pharm. Sci., 2000, 11, S81-S91.

52 C. A. Stine and J. M. Munson, Front. Oncol., 2019, 9, 966.

53 S. Aftab, A. Shah, A. Nadhman, S. Kurbanoglu, S. A. Ozkan, D. D. Dionysiou, S. S. Shukla and T. M. Aminabhavi, Int. J. Pharm., 2018, 540, 132-149.

54 A. K. Iyer, G. Khaled, J. Fang and H. Maeda, Drug Discovery Today, 2006, 11, 812-818.

55 M. A. Khiavi, A. Safary, J. Barar, A. Ajoolabady, M. H. Somi and Y. Omidi, Cell. Mol. Life Sci., 2020, 77, 997-1019.

56 F. S. Anarjan, Nano-Struct. Nano-Objects, 2019, 19, 100370.

57 H. Choudhury, M. Pandey, P. X. Chin, Y. L. Phang, J. Y. Cheah, S. C. Ooi, K.-K. Mak, M. R. Pichika, P. Kesharwani and Z. Hussain, Drug Delivery Transl. Res., 2018, 8, 1545-1563.
58 G. P. Adams, R. Schier, A. M. McCall, H. H. Simmons, E. M. Horak, R. K. Alpaugh, J. D. Marks and L. M. Weiner, Cancer Res., 2001, 61, 4750-4755.

59 S. Gosk, T. Moos, C. Gottstein and G. Bendas, Biochim. Biophys. Acta, Biomembr., 2008, 1778, 854-863.

60 S. S. Kelkar and T. M. Reineke, Bioconjugate Chem., 2011, 22, 1879-1903.

61 R. Weinberg and D. Hanahan, Cell, 2000, 100, 57-70.

62 P. P. Hsu and D. M. Sabatini, Cell, 2008, 134, 703-707.

63 C. H. Chung, K. Ely, L. McGavran, M. Varella-Garcia, J. Parker, N. Parker, C. Jarrett, J. Carter, B. A. Murphy and J. Netterville, J. Clin. Oncol., 2006, 24, 4170-4176.

64 P. M. Pereira, L. Abma, K. E. Henry and J. S. Lewis, Cancer Lett., 2018, 419, 139-151.

65 M. Li, L. Zhang, W. Tang, J.-C. Duan, Y.-J. Jin, L.-L. Qi and N. Wu, Cancer Imaging, 2019, 19, 1-11.

66 A. Girigoswami, W. Yassine, P. Sharmiladevi, V. Haribabu and K. Girigoswami, Sci. Rep., 2018, 8, 16459.

67 D.-E. Lee, H. Koo, I.-C. Sun, J. H. Ryu, K. Kim and I. C. Kwon, Chem. Soc. Rev., 2012, 41, 2656-2672.

68 W. Dong, Y. Li, D. Niu, Z. Ma, J. Gu, Y. Chen, W. Zhao, X. Liu, C. Liu and J. Shi, Adv. Mater., 2011, 23, 5392-5397.

69 X. Huang, Z. Yin, S. Wu, X. Qi, Q. He, Q. Zhang, Q. Yan, F. Boey and H. Zhang, Small, 2011, 7, 1876-1902.

70 J. Yang, J. Choi, D. Bang, E. Kim, E. K. Lim, H. Park, J. S. Suh, K. Lee, K. H. Yoo and E. K. Kim, Angew. Chem., Int. Ed., 2011, 50, 441-444.

71 P. Zhang, J. Yang, W. Li, W. Wang, C. Liu, M. Griffith and W. Liu, J. Mater. Chem., 2011, 21, 7755-7764.

72 J. Yang, C. H. Lee, H. J. Ko, J. S. Suh, H. G. Yoon, K. Lee, Y. M. Huh and S. Haam, Angew. Chem., Int. Ed., 2007, 46, 8836-8839.

73 E. K. Lim, Y. M. Huh, J. Yang, K. Lee, J. S. Suh and S. Haam, Adv. Mater., 2011, 23, 2436-2442.

74 C. H. Lee, S. H. Cheng, Y. J. Wang, Y. C. Chen, N. T. Chen, J. Souris, C. T. Chen, C. Y. Mou, C. S. Yang and L. W. Lo, Adv. Funct. Mater., 2009, 19, 215-222.

75 E.-K. Lim, J. Yang, C. P. Dinney, J.-S. Suh, Y.-M. Huh and S. Haam, Biomaterials, 2010, 31, 9310-9319.

76 K. Kim, J. H. Kim, H. Park, Y.-S. Kim, K. Park, H. Nam, S. Lee, J. H. Park, R.-W. Park and I.-S. Kim, J. Controlled Release, 2010, 146, 219-227.

77 T. Nam, S. Park, S.-Y. Lee, K. Park, K. Choi, I. C. Song, M. H. Han, J. J. Leary, S. A. Yuk and I. C. Kwon, Bioconjugate Chem., 2010, 21, 578-582.

78 S. Santra, C. Kaittanis and J. M. Perez, Mol. Pharmaceutics, 2010, 7, 1209-1222.

79 S. Biffi, R. Voltan, B. Bortot, G. Zauli and P. Secchiero, Expert Opin. Drug Delivery, 2019, 16, 481-496.

80 X. L. Hu, N. Kwon, K. C. Yan, A. C. Sedgwick, G. R. Chen, X. P. He, T. D. James and J. Yoon, Adv. Funct. Mater., 2020, 30, 1907906.

81 S. H. Nam, Y. M. Bae, Y. I. Park, J. H. Kim, H. M. Kim, J. S. Choi, K. T. Lee, T. Hyeon and Y. D. Suh, Angew. Chem., Int. Ed., 2011, 50, 6093-6097. 
82 H. Y. Yoon, H. Koo, K. Y. Choi, S. J. Lee, K. Kim, I. C. Kwon, J. F. Leary, K. Park, S. H. Yuk and J. H. Park, Biomaterials, 2012, 33, 3980-3989.

83 H. B. Na, I. C. Song and T. Hyeon, Adv. Mater., 2009, 21, 2133-2148.

84 S. Rojas, A. Arenas-Vivo and P. Horcajada, Coord. Chem. Rev., 2019, 388, 202-226.

85 K. Lu, T. Aung, N. Guo, R. Weichselbaum and W. Lin, Adv. Mater., 2018, 30, 1707634.

86 T. Kim, E. J. Cho, Y. Chae, M. Kim, A. Oh, J. Jin, E. S. Lee, H. Baik, S. Haam and J. S. Suh, Angew. Chem., Int. Ed., 2011, 50, 10589-10593.

87 V. Haribabu, A. S. Farook, N. Goswami, R. Murugesan and A. Girigoswami, J. Biomed. Mater. Res., Part B, 2016, 104, 817-824.

88 P. Sharmiladevi, N. Akhtar, V. Haribabu, K. Girigoswami, S. Chattopadhyay and A. Girigoswami, ACS Appl. Bio Mater., 2019, 2, 1634-1642.

89 Y. Zhao, L. N. Lin, Y. Lu, S. F. Chen, L. Dong and S. H. Yu, Adv. Mater., 2010, 22, 5255-5259.

90 J. Gao, G. Liang, B. Zhang, Y. Kuang, X. Zhang and B. Xu, J. Am. Chem. Soc., 2007, 129, 1428-1433.

91 M. Jeyaraj, S. Gurunathan, M. Qasim, M.-H. Kang and J.-H. Kim, Nanomaterials, 2019, 9, 1719.

92 S. Kaida, H. Cabral, M. Kumagai, A. Kishimura, Y. Terada, M. Sekino, I. Aoki, N. Nishiyama, T. Tani and K. Kataoka, Cancer Res., 2010, 70, 7031-7041.

93 R. Kopelman, Y.-E. L. Koo, M. Philbert, B. A. Moffat, G. R. Reddy, P. McConville, D. E. Hall, T. L. Chenevert, M. S. Bhojani and S. M. Buck, J. Magn. Magn. Mater., 2005, 293, 404-410.

94 M. Vimaladevi, K. C. Divya and A. Girigoswami, J. Photochem. Photobiol. B, 2016, 162, 146-152.

95 T. Schluep, J. Hwang, I. J. Hildebrandt, J. Czernin, C. H. J. Choi, C. A. Alabi, B. C. Mack and M. E. Davis, Proc. Natl. Acad. Sci. U. S. A., 2009, 106, 11394-11399.

96 H. P. Luehmann, E. D. Pressly, L. Detering, C. Wang, R. Pierce, P. K. Woodard, R. J. Gropler, C. J. Hawker and Y. Liu, J. Nucl. Med., 2014, 55, 629-634.

97 C.-T. Yang, K. K. Ghosh, P. Padmanabhan, O. Langer, J. Liu, D. N. C. Eng, C. Halldin and B. Gulyás, Theranostics, 2018, 8, 6210.

98 Y. Shen, M. Li, T. Liu, J. Liu, Y. Xie, J. Zhang, S. Xu and H. Liu, Int. J. Nanomed., 2019, 14, 4029.

99 Y. Liu, J. Li, M. Chen, X. Chen and N. Zheng, Theranostics, 2020, 10, 10057.
100 D. Zhi, T. Yang, J. O'hagan, S. Zhang and R. F. Donnelly, J. Controlled Release, 2020, 325, 52-71.

101 A. B. E. Attia, G. Balasundaram, M. Moothanchery, U. Dinish, R. Bi, V. Ntziachristos and M. Olivo, Photoacoustics, 2019, 16, 100144.

102 X. Li, Z. Cai, L.-P. Jiang, Z. He and J.-J. Zhu, Bioconjugate Chem., 2019, 31, 332-339.

103 A. Kamrani, A. Mehdizadeh, M. Ahmadi, L. AghebatiMaleki and M. Yousefi, Expert Opin. Ther. Targets, 2019, 23, 447-456.

104 M. E. Davis, J. E. Zuckerman, C. H. J. Choi, D. Seligson, A. Tolcher, C. A. Alabi, Y. Yen, J. D. Heidel and A. Ribas, Nature, 2010, 464, 1067.

105 Y. G. Shellman, W. R. Howe, L. A. Miller, N. B. Goldstein, T. R. Pacheco, R. L. Mahajan, S. M. LaRue and D. A. Norris, J. Invest. Dermatol., 2008, 128, 949-956.

106 K. Hayashi, K. Ono, H. Suzuki, M. Sawada, M. Moriya, W. Sakamoto and T. Yogo, Chem. Mater., 2010, 22, 3768-3772.

107 Y. Cheng, J. D. Meyers, A.-M. Broome, M. E. Kenney, J. P. Basilion and C. Burda, J. Am. Chem. Soc., 2011, 133, 2583-2591.

108 H.-Y. Niu, W.-H. Li, Y.-L. Shi and Y.-Q. Cai, Chem. Commun., 2011, 47, 4454-4456.

109 A. Liberman, H. P. Martinez, C. N. Ta, C. V. Barback, R. F. Mattrey, Y. Kono, S. L. Blair, W. C. Trogler, A. C. Kummel and Z. Wu, Biomaterials, 2012, 33, 5124-5129.

110 E.-K. Lim, E. Jang, B. Kim, J. Choi, K. Lee, J.-S. Suh, Y.-M. Huh and S. Haam, J. Mater. Chem., 2011, 21, 12473-12478.

111 H. Maeda, Bioconjugate Chem., 2010, 21, 797-802.

112 M. M. Yallapu, S. P. Foy, T. K. Jain and V. Labhasetwar, Pharm. Res., 2010, 27, 2283-2295.

113 K. McKeage and C. M. Perry, Drugs, 2002, 62, 209-243.

114 M. H. Cohen, Y. L. Shen, P. Keegan and R. Pazdur, The oncologist, 2009, 14, 1131-1138.

115 R. M. Kong, X. B. Zhang, Z. Chen and W. Tan, Small, 2011, 7, 2428-2436.

116 Y. Tsutsui, K. Tomizawa, M. Nagita, H. Michiue, T.-I. Nishiki, I. Ohmori, M. Seno and H. Matsui, J. Controlled Release, 2007, 122, 159-164.

117 M. Semmler-Behnke, W. G. Kreyling, J. Lipka, S. Fertsch, A. Wenk, S. Takenaka, G. Schmid and W. Brandau, Small, 2008, 4, 2108-2111. 